\title{
Clinical and surgical outcomes of 3 cycles versus 6 cycles of neoadjuvant chemotherapy in advanced ovarian carcinoma
}

\author{
Monika Thakur¹, Archana Kumari²*, S. C. Saha ${ }^{2}$
}

\begin{abstract}
${ }^{1}$ Department of Obstetrics and Gynecology, DYSPGMC, Nahan, Sirmaur, Himachal Pradesh, India
${ }^{2}$ Department of Obstetrics and Gynecology, PGIMER, Chandigarh, India
\end{abstract}

Received: 27 September 2019

Accepted: 31 October 2019

\section{*Correspondence:}

Dr. Archana Kumari,

E-mail:07arch@gmail.com

Copyright: ( $)$ the author(s), publisher and licensee Medip Academy. This is an open-access article distributed under the terms of the Creative Commons Attribution Non-Commercial License, which permits unrestricted non-commercial use, distribution, and reproduction in any medium, provided the original work is properly cited.

\section{ABSTRACT}

Background: Epithelial cancers are the most common ovarian malignancy accounting for $90 \%$ of all type of ovarian cancer. Objective of this study was to evaluate the surgical morbidity and to study clinical outcomes of 3 cycles versus 6 cycles of neoadjuvant chemotherapy.

Methods: A total 30 women with diagnosis of advanced epithelial ovarian cancer were randomly divided equally to receive either 3 cycles (Group 1) or 6 cycles (Group 2) of neoadjuvant chemotherapy. End points noted were duration of surgery, extent of surgery, perioperative complications and length of stay.

Results: Both groups had comparable demographic profile. Surgery was more difficult in early IDS group with lesser percentage of patients achieving minimum intended surgery in early IDS (61.53\% versus $80.47 \%)$ peri-operative complications were seen more commonly in early IDS group compared to late IDS group (26.66\% versus $69.2 \%$ ). However mean duration of surgery was similar in both groups. Mean duration of stay in hospital was 5.4 days in late IDS group and 7.6 days in early IDS group. The mean follow-up period in late IDS was 7 months and in early IDS was 5 months. Survival in both groups when patients were followed up till the end of study period (12 months) was similar $(\mathrm{p}=0.186)$.

Conclusions: Authors conclude that late IDS may be used as a treatment option in the high-risk group of patients. Though there was no significant difference at 6 months follow up, higher percent of women were disease free in late IDS.

Keywords: Advanced ovarian carcinoma, Epithelial ovarian cancer, Early interval debulking surgery, Late interval debulking surgery, Neoadjuvant chemotherapy

\section{INTRODUCTION}

Epithelial cancers are the most common ovarian malignancy accounting for $90 \%$ of all type of ovarian cancer. Approximately $70 \%$ of women have advanced stage III or IV carcinoma ovary at the time of diagnosis as it has vague and nonspecific symptoms in early stage of disease. One of the treatment options in advanced ovarian cancer is interval debulking surgery following neoadjuvant chemotherapy (NACT). Patients characterized by high initial tumor dissemination, stage IV disease, poor performance or nutritional status, age $\geq 75$ years with high surgical complexity score, achieving low residual disease was associated with morbidity of $63.6 \%$ and limited survival benefit. ${ }^{1}$ NACT in such patients is associated with increased optimal cytoreduction with less morbidity. ${ }^{2}$ Advantages of neoadjuvant chemotherapy include an increased rate of optimal debulking, less extensive surgery, reduced blood loss, lower morbidity, shortened hospital stay, and improved quality of life. ${ }^{3}$ The current recommendation is to perform interval debulking surgery early, after three cycles of NACT. In recent period the concept of late IDS (interval debulking surgery) has been introduced and it 
has been seen that 6 cycles before surgery may be better. This change was motivated by the feeling that surgery was easier at late IDS.

Thus, this study was planned to evaluate the optimal timing of IDS and test the hypothesis that late interval debulking surgery after 6 cycles of neoadjuvant chemotherapy will improve the surgical outcomes.

\section{METHODS}

This prospective randomized controlled study was conducted in the department of obstetrics and gynaecology and the department of radiotherapy in collaboration with the department of cytology and gynecological pathology of the post-graduate institute of medical education and research, Chandigarh from July 2012 to December 2013.

\section{Inclusion criteria}

- Thirty women with primary epithelial ovarian cancer, fallopian tube cancer and primary peritoneal carcinomatosis who were referred to radiotherapy department for neoadjuvant chemotherapy were included in this study

- Diagnosis of ovarian carcinoma was made on basis of ovarian mass, raised CA125 level and fine needle aspiration cytology report

- Diagnosis of primary peritoneal carcinomatosis was established by increased CA125 level, positive fluid cytology, normal CEA and CA19.9 level, normal upper and lower gastrointestinal endoscopy, normal mammography and ultrasound and CT scan report to rule out other abdominal carcinomas

- Stage IIIc or IV cancer based on clinical, radiological and sonological findings.

\section{Exclusion criteria}

- Presence histological types of ovarian cancers other than EOC

- Patients already received chemotherapy or surgery outside

- $\quad$ Patients with unknown primary

- Serious disabling disease that would contraindicate chemotherapy

- ECOG performance status greater than 2

- Patients not willing for trial.

The It was a pilot study and a total of 30 patients with ovarian epithelial carcinoma stage III or IV who were referred to oncology department for neoadjuvant chemotherapy were involved. Decisions for NACT were taken by senior gynaecologist of three clinical units of department of OBG in PGIMER.

The randomization was done by computer generated randomized table. Patients were randomized into 2 groups:
- Group 1 Late IDS: 6 cycles of NACT

- Group 2 Early IDS: 3 cycles of NACT

For each patient detailed history was taken. Clinical findings were noted. Surgery was performed with intention to do total abdominal hysterectomy with $\mathrm{B} / \mathrm{L}$ salpingoophorectomy, infracolic omentectomy with maximal attempt for cytoreduction to no residual disease without causing unacceptable morbidity to patient. Follow up of patients with CA-125 level and if required CT scan was done at the end of treatment.

\section{End points studied were}

- Duration of surgery

- Intra /post op blood transfusion requirement

- Bowel/bladder/vessel injury

- No of days of hospitalization

- Postoperative complication.

\section{Statistical analysis}

The statistical analysis was carried out using Statistical Package for Social Sciences (SPSS Inc., Chicago, IL, version 15.0 for Windows). Mean and medians was calculated for all quantitative variables and for measures of dispersion standard deviation or standard error were calculated. Normality of data was checked by measures of Kolmogorov Smirnov tests of normality. For normally distributed data means of two groups was compared using t-test. For skewed data Mann -Whitney test was applied. Qualitative or categorical variables were described as frequencies and proportions. Proportions were compared by using Chi square or Fisher's exact test whichever was applicable. All statistical tests were two-sided and was performed at a significance level of $\alpha=.05$.

\section{RESULTS}

Both the groups had comparable demographic profile. Table 1 shows mode of diagnosis in 30 patients recruited in current study. Table 2 shows indication of neoadjuvant chemotherapy and type of surgery done in both the groups. Two patients died in early IDS group within 1 month of receiving $1^{\text {st }}$ chemotherapy in spite of the fact that their performance status was 2. Probable cause of death was thromboembolism.

As shown in Table 3, twenty six percent of patients in Group 1 and $46.15 \%$ of patients in Group 2 required blood transfusions in peri-operative period. In Group 2, $7.6 \%$ had enterostomy and serosal tear and same was repaired, $7.6 \%$ had haematuria, and another $7.6 \%$ had burst abdomen on $7^{\text {th }}$ postoperative day. Patient was admitted in ward for 20 days. Resuturing was done on $21^{\text {st }}$ day. It was observed that Group 2 had more perioperative complications though it was not statistically significant $(\mathrm{p}=0.061)$. Mean duration of surgery in Group 1 was $2.3 \pm 0.73$ and in group 2 was $2.23 \pm 0.56$ hours 
which was comparable $(\mathrm{p}=.065)$. Mean follow up period in Group 1 was $7.006 \pm 3.731$ months with a range of 2-12 months and in Group 2 were 5.079 \pm 2.326 months with a range of 2-8 months. Follow up period of $\geq 6$ months was seen in 10 patients in Group 1 and 8 patients in Group 2.
A survival curve was drawn as shown in figure 1. The overall survival in both groups when patients were followed for a period of 12 months was similar $(\mathrm{P}=0.186$ by $\log$ rank test).

Table 1: Mode of cytological diagnosis.

\begin{tabular}{|llll|}
\hline & FNAC & Positive cytology (pleural/peritoneal) & FNAC+ positive cytology \\
\hline Group 1 (6 cycles) & 3 & $9(5$ peritoneal+ 4 pleural) & 3 \\
\hline Group 2 (3 cycles) & 5 & $3(2$ peritoneal+ 1 pleural $)$ & 7 \\
\hline Total & $\mathbf{8}$ & $\mathbf{1 2}$ & $\mathbf{1 0}$ \\
\hline
\end{tabular}

Table 2: Indication of neoadjuvant chemotherapy and type of surgery.

\begin{tabular}{|lll|}
\hline Characteristics & Group 1, N=15 & Group 2, N=13 P value \\
\hline Indication of neoadjuvant chemotherapy & & \\
\hline Stage IV disease excluding pleural cytology & $1(6.6 \%)$ & $3(20 \%)$ \\
\hline Metastasis at site where resection is difficult & $5(33.33 \%)$ & $7(46.66 \%)$ \\
\hline Hard fixed pelvic mass & 0 & $1(6.6 \%)$ \\
\hline Numerous peritoneal deposits or plaque or large omental cake & $9(60 \%)$ & $4(26.66 \%)$ \\
\hline Type of surgery & & \\
\hline TAH + BSO + Ometenectomy & $13(80.47 \%)$ & $8(61.53 \%)$ \\
\hline BSO+Peritoneal/omental biopsy & $1(6.6 \%)$ & $4(30.76 \%)$ \\
\hline
\end{tabular}

Table 3. Perioperative complications, duration of surgery, length of stay and follow-up.

\begin{tabular}{|c|c|c|c|}
\hline Characteristics & Group 1, N=15 & Group 2, N=13 & P value \\
\hline \multicolumn{4}{|l|}{ Perioperative complications } \\
\hline Blood transfusion & $4(26.66 \%)$ & $6(46.15 \%)$ & \multirow{4}{*}{0.061} \\
\hline Bowel injury & 0 & $1(7.6 \%)$ & \\
\hline Burst abdomen & 0 & $1(7.6 \%)$ & \\
\hline Haematuria & 0 & $1(7.6 \%)$ & \\
\hline Duration of surgery (hours) & $2.3 \pm 0.73$ & $2.23 \pm 0.56$ & 0.065 \\
\hline Hospital stay & $5.4 \pm 2.06$ & $7.6 \pm 3.86$ & 0.595 \\
\hline Follow up & $7.006 \pm 3.731$ & $5.079 \pm 2.326$ & \\
\hline \multicolumn{4}{|c|}{ Disease status during follow-up } \\
\hline Disease free & $7(46.66 \%)$ & $9(69.2 \%)$ & \multirow{4}{*}{0.228} \\
\hline Persistent & $8(53.33 \%)$ & $4(30.7 \%)$ & \\
\hline Recurrence & 0 & 0 & \\
\hline Progression & 0 & 0 & \\
\hline \multicolumn{4}{|c|}{ Disease status during 6 months of follow-up } \\
\hline Disease free & 7 & 7 & \multirow[t]{2}{*}{0.588} \\
\hline Persistent & 3 & 1 & \\
\hline
\end{tabular}

\section{DISCUSSION}

Ovarian carcinoma is the $2^{\text {nd }}$ most common gynecological cancer in India. ${ }^{4}$ It represents the greatest clinical challenge because of its high mortality. One of the treatment options in advanced ovarian cancer is interval debulking surgery following neo adjuvant chemotherapy (NACT). NACT in such patients is associated with increased optimal cytoreduction with less morbidity. ${ }^{2}$ The current recommendation is to perform interval debulking surgery early, after three cycles of NACT. ${ }^{5}$ In recent period the concept of late IDS (interval debulking surgery) has been introduced and it has been seen that 6 cycles before surgery may be better option. ${ }^{6}$ This change was motivated by the feeling that surgery was easier at late IDS and higher optimal resection rates were obtained at late IDS in comparison to earlier IDS. No study in the past has compared early IDS with late IDS in a 
prospective way. The present study was planned to evaluate the optimal timing of IDS and test the hypothesis that late interval debulking surgery after 6 cycles of neo adjuvant chemotherapy will improve the survival along with reduction in surgical morbidity.

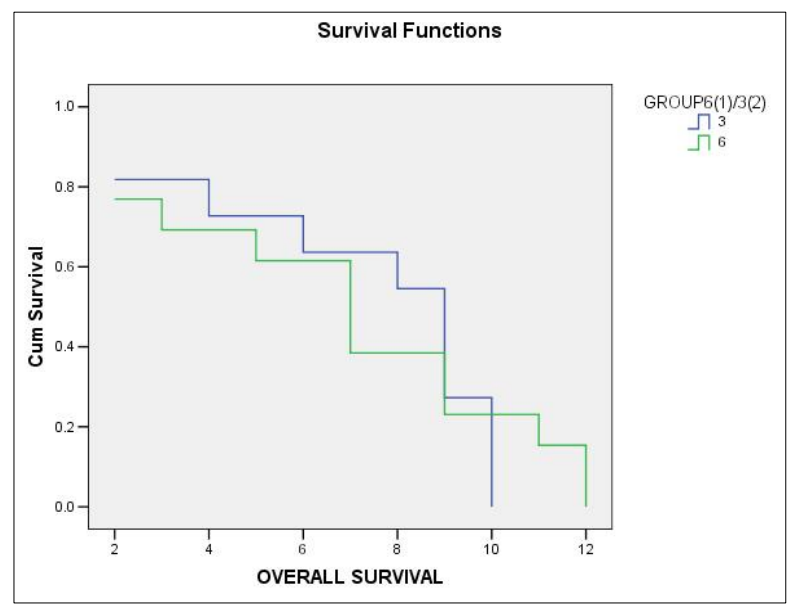

Figure 1: Overall survival for patients with stage III/IV ovarian cancer in the 3 cycles versus 6 cycles of neoadjuvant chemotherapy.

In the current study the diagnosis of ovarian, fallopian tube and peritoneal carcinoma was made by FNAC from ovarian and other metastatic mass, cytological examination of ascitic or pleural fluid, CT scan examination and serum tumor markers like CA125. Vergote et al, in their study used biopsy to diagnose ovarian carcinoma. ${ }^{5}$ If biopsy was not available, FNAC along with raised serum CA125, CA125/ CEA (>25), pelvic mass and upper abdominal mass more than $2 \mathrm{~cm}$, peritoneal fluid cytology in absence of primary in gastrointestinal tract and breast was accepted. Glasgow et al used pelvic mass, with biopsy or cytology specimen showing carcinoma with evidence of stage IV disease, as evidence of ovarian cancer. ${ }^{7}$ In another study by Deo et al diagnosis of ovarian carcinoma was established by CECT of abdomen, ascitic fluid cytology and CA125 level in $75 \%$ of patients and by laparotomy in $25 \%$ of patients. ${ }^{8}$ Diagnostic accuracy of FNAC in diagnosing ovarian carcinoma has shown to be up to $90-95 \%$ in various studies. ${ }^{9-11}$ Accuracy of fluid cytology has been shown to be more than $80 \%$ in a study by Cefis et al. ${ }^{12}$

Abdominal distension was most common presenting symptom followed by loss of appetite. Weight loss, bowel and bladder symptoms were also seen commonly in both groups. In a study by Goff et al $70 \%$ of patients had abdominal or gastrointestinal symptoms, $58 \%$ had pain, $34 \%$ had urinary symptoms whereas $26 \%$ of patients presented with pelvic discomfort. ${ }^{13}$ The findings were similar to that in the current study. Hypertension was the most common co morbidity seen in both groups. None had serious disabling disease which would contraindicate primary platinum-based chemotherapy. A total $26 \%$ of patients in late IDS group and $20 \%$ of women in early IDS group had history of cancer in family. However, none had family history of ovarian or breast cancer which is seen in hereditary ovarian cancer associated with mutation in BRCA1 gene.

The basic principle behind all the criteria for selecting patients for NACT was, when the standard surgical approach deemed incapable of achieving optimal cytoreduction with acceptable morbidity. In accordance with these guidelines in our study we chose patients for NACT either when the metastatic tumor was at an unresectable site, or was of very large volume, was stage IV disease or was of diffuse nature so as to preclude optimal cytoreduction. In the current study, in late IDS group, $6.6 \%$ patient received NACT for metastasis in liver and lung, in $33.33 \%$ cases NACT was given for metastasis at sites where resection was difficult. In $60 \%$ of patients there were numerous peritoneal deposits or plaque or very large omental cake. In early IDS group, $20 \%$ patients received NACT for metastasis to liver and lung, $46.66 \%$ had metastasis at site where resection was difficult. Numerous peritoneal deposits or large omental caking was present in $26.66 \%$ of patients. One patient in early IDS was referred for NACT as it was felt clinically and radiologically that mass was not resectable by standard surgery which was later corroborated by intra operative findings.

In the current study the minimum intended surgery was removal of primary tumor (Bilateral salpingooophorectomy), uterus and omentum with maximal attempt for cytoreduction. This minimum intended surgery could not be achieved in $30.76 \%$ of patients in early IDS compared to $6.6 \%$ in late IDS group. Hysterectomy was not possible in these patients due to dense adhesions between uterus and surrounding structure like bowel and bladder. Surgery was more difficult in early IDS group compared to late IDS group with lesser percentage of patients achieving minimum intended surgery in early IDS (61.53\% versus $80.47 \%)$. It was also observed that increased need of help from general surgeons was required in early IDS group in comparison to late IDS group (23.07\% versus $13.33 \%$ in early and late IDS respectively).

In the present study peri-operative complications were seen more commonly in early IDS group compared to late IDS group (26.66\% versus $69.2 \%$ ). Blood transfusion was required in $46.66 \%$ of patients in early IDS group compared to $26.66 \%$ in late IDS group. Other complications like bowel injury, haematuria, burst abdomen was seen more commonly in early IDS group. However, in study by Stoeckle et al significant morbidity, defined by needing prolonged or new hospitalization or reoperation, was similar between the 2 groups (15 versus $13 \%) .^{6}$

The mean duration of surgery and hospital stay was similar between two groups in the current study. The mean duration of surgery in early IDS group was 
$2.2 \pm 0.56$ hours and in late IDS group was $2.3 \pm 0.73$ hours The mean duration of hospital stay in late IDS group was $5.4 \pm 2.06$ days and in in early IDS group was 7.6 \pm 3.86 days.

Mean follow up period in late IDS group was $7.006 \pm 3.731$ months with a range of 2-12 months and in early IDS group was $5.079 \pm 2.326$ months with a range of 2-8 months. Patients who were followed up for a period $\geq$ 6 month after completion of their treatment, higher percentage of women in late IDS were found to be disease free compared to that in early IDS $(70 \%$ versus $66.66 \%$ ) $20 \%$ in late IDS and $33.33 \%$ in early IDS had persistent disease and $10 \%$ had progression in late IDS group. Survival in both groups when patients were followed up till the end of study period (12 months) was similar $(\mathrm{p}=0.186)$.

\section{CONCLUSION}

We conclude that late IDS may be used as a treatment option in the high-risk group of patients. Administering 6 cycles of neoadjuvant chemotherapy prior to debulking surgery helps in achieving lesser surgical morbidity and decreased perioperative complications along with reduced length of stay in the hospital though the difference did not attain statistical significance. Though there was no significant difference between both the groups at 6-month follow up, higher percent of women were disease free in late IDS.

\section{ACKNOWLEDGMENTS}

Authors would like to thank institute, patients and coauthors who helped in completing this article successfully. This research did not receive any specific grant from funding agencies in the public, commercial, or not-for-profit sectors. No writing assistance was taken for writing the above manuscript.

Funding: No funding sources Conflict of interest: None declared

Ethical approval: The study was approved by the Institutional Ethics Committee

\section{REFERENCES}

1. Aletti GD, Eisenhower EL, Santillan A, Axtell A, Aletti A, Chi SD. Identification of patient groups of highest risks from traditional approach to ovarian cancer treatment. Gynecol Oncol. 2011;120:23-8.

2. Lee SJ, Kim BG, Lee JW. Preliminary results of neoadjuvant chemotherapy with paclitaxel and cisplatin in patients with advanced epithelial ovarian cancer who are inadequate for optimum primary surgery. J Obstet Gynecol. 2006;32(1):99-106.

3. Baekelandt M. The potential role of neoadjuvant chemotherapy in advanced ovarian cancer. Int $\mathbf{J}$ Gynecol Cancer. 2003;13:163-8.

4. Consolidated report of population-based cancer registaries 2001-2004. National cancer registry program. Indian council of medical research. Bangalore: 2006.

5. Vergote CG, Trope F, Amant GB. EORTCGCG/NCIC-CTG randomized trial comparing primary debulking surgery with neoadjuvant chemotherapy in stage III C -IV ovarian, fallopian tube and peritoneal cancer. Plenary presentation at the $12^{\text {th }}$ Biennial meeting. International Gynecologic Cancer Society IGCS, Bangkok, Thailand October; 2008(abs.); 2008:25-28.

6. Stoeckle E, Boubli B, Floquet A, Brouste V, Sire M, Croce $\mathrm{S}$, et al. Optimal timing of interval debulking surgery in advanced ovarian cancer: yet to be defined? Eu J Obstet Gynecol Repro Biol. 2011;159:407-12.

7. Glasgow MA, Yu H, Rutherford TJ, Azodi M, Silasi DA, Santi AD, et al. Neoadjuvant chemotherapy is an effective way of managing elderly women with advanced stage ovarian cancer (FIGO stage IIIc and IV). J Surg Oncol. 2013;107:195-200.

8. Deo SV, Goyal H, Shukla NK. Neoadjuvant chemotherapy followed by surgical cytoreduction in advanced epithelial ovarian cancer. Indian J Cancer. 2006;43(3):117-21.

9. Pajtler M, Cugalj B, Milojković M, Makarović Z. The importance of directed cytological examinations in the diagnosis of ovarian tumors. Jugosl Ginekol Perinatol. 1989;29(3-4):133-6.

10. Sood T, Handa U, Mohan H, Goel P. Evaluation of aspiration cytology of ovarian masses with histopathological correlation. Cytopathol. 2010;21(3):176-85.

11. Geir G, Kraus H, Schuhman R. Aspiration biopsy in ovarian tumors. Geburtshilfe Frauenheilked. 1975;35(1):48-54.

12. Cefis F, Forni A, Carinelli S. Significance of acsitic fluid and peritoneal washing in ovarian tumor diagnosis. Tumori. 1978;64(1):77-8.

13. Goff BA, Mandel LS, Muntz HG, Melancon CH. Ovarian cancer diagnosis: results of a national ovarian cancer survey. Cancer. 2000;89:2068-75.

Cite this article as: Thakur M, Kumari A, Saha SC. Clinical and surgical outcomes of 3 cycles versus 6 cycles of neoadjuvant chemotherapy in advanced ovarian carcinoma. Int J Reprod Contracept Obstet Gynecol 2019;8:4985-9. 